\title{
Anaesthesia Challenges during Sternal Wound Debridement
}

\author{
K. G. Kritika ${ }^{1}$ Poonam Malhotra Kapoor ${ }^{2}$ Minati Choudhury ${ }^{2}$
}

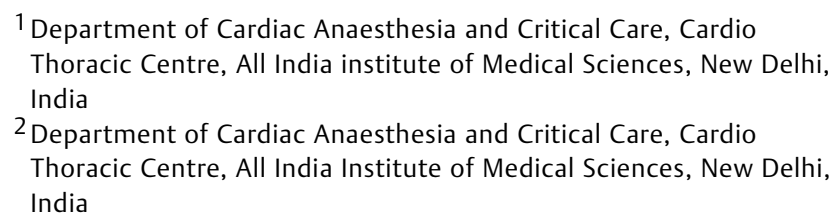

2 Department of Cardiac Anaesthesia and Critical Care, Cardio Thoracic Centre, All India Institute of Medical Sciences, New Delhi, India

J Card Crit Care 2021;5:238-241.

\begin{abstract}
Address for correspondence Poonam Malhotra Kapoor, MD, DNB, Department of Cardiac Anaesthesia and Critical Care, Cardio Thoracic Centre, All India Institute of Medical Sciences, New Delhi, 110029, India (e-mail: drpoonamaiims@gmail.com).
\end{abstract}

\begin{abstract}
Keywords

- airway management

- mitral valve replacement

- sternal wound debridement

A 50-year-old female weighing $80 \mathrm{~kg}$ is posted for a deep sternal wound debridement, post mitral valve replacement (MVR), and post-CABG (Coronary Artery Bypass Grafting) (left internal mammary artery (LIMA) $\rightarrow$ left anterior descending (LAD)). She had sternal osteomyelitis and had wound debridement thrice before. She had also history of cardiac injury repair during second wound debridement.

Her initial three tissue cultures showed Acinetobacter positive. Her fourth tissue culture shows Staphylococcus haemolyticus positive. Histopathology shows necrotizing granuloma. Two-dimensional Echocardiography (ECHO) shows post-MVR, no Mitral regurgitation (MR), mild Tricuspid Regurgitation (TR) (right ventricular systolic pressure (RVSP)-24 + Retrograde autologous priming (RAP)), Inferior Vena Cava \#x0028;IVC) $1.6 \mathrm{~cm}$ with respiratory collapse, no pericardial effusion, no Left atrium (LA) clot/veg, and normal biventricular function.
\end{abstract}

\section{Introduction}

A 50 -year-old female weighing $80 \mathrm{~kg}$ is posted for a deep sternal wound debridement, post mitral valve replacement (MVR), and post-CABG (LIMA $\rightarrow$ LAD). She had sternal osteomyelitis and had wound debridement thrice before. She had also history of cardiac injury repair during second wound debridement.

Her initial three tissue cultures showed Acinetobacter positive. Her fourth tissue culture shows Staphylococcus haemolyticus positive. Histopathology shows necrotizing granuloma. Two-dimensional ECHO shows post-MVR, no MR, mild TR (RVSP-24+RAP), IVC $1.6 \mathrm{~cm}$ with respiratory collapse, no pericardial effusion, no LA clot/veg, and normal biventricular function.

The patient was planned for sternal wound debridement under general anesthesia. She was on oxygen face mask with $2 \mathrm{~L} / \mathrm{m}$ of oxygen. She had the right internal jugular vein, central venous catheter in situ, and an $18 \mathrm{G}$ peripheral line in her left hand. Her vitals were stable without any support.

The intraoperative hemodynamic monitors used were Non-invasive Blood Pressure (NIBP), electrocardiogram (ECG), pulse oximeter, BiSpecteral Index (BIS), urine output, and capnography. She was induced with inj. propofol $100 \mathrm{mg}$, inj. fentanyl $50 \mathrm{mcg}$, inj. midazolam $1 \mathrm{mg}$, and the airway was secured with a ProSeal LMA size 3. Anesthesia was
DOI https://doi.org/ 10.1055/s-0041-1742244. ISSN 2457-0206.
(C) 2022. Official Publication of The Simulation Society (TSS), accredited by International Society of Cardiovascular Ultrasound (ISCU). All rights reserved.

This is an open access article published by Thieme under the terms of the Creative Commons Attribution-NonDerivative-NonCommercial-License, permitting copying and reproduction so long as the original work is given appropriate credit. Contents may not be used for commercial purposes, or adapted, remixed, transformed or built upon. (https://creativecommons.org/ licenses/by-nc-nd/4.0/)

Thieme Medical and Scientific Publishers Pvt. Ltd., A-12, 2nd Floor, Sector 2, Noida-201301 UP, India 
maintained with oxygen, air, and sevoflurane. Wound debridement and dressing were done. Vitals were stable throughout the procedure and it was uneventful.

During reversal, inj. neostigmine $2.5 \mathrm{mg}$ with glycopyrrolate $0.4 \mathrm{mg}$ was given. Inj. calcium gluconate $1 \mathrm{~g}$ intravenous (IV) was given as pressures were low. BIS was 40 and the reversal was inadequate. Additional inj. neostigmine $1 \mathrm{mg}$ was given. The patient had poor respiratory effort. Infusion noradrenalin was started at $0.05 \mathrm{mcg} / \mathrm{kg} / \mathrm{min}$ since she had hypotension. She then had hypercarbia and desaturation and she was intubated with cuffed endotracheal tube (ETT) size 7.5 at fixed 21 mark. Her saturation picked up and was shifted to intensive care unit (ICU) being intubated, wherein inj. noradrenalin was started and she remained hemodynamically stable. She was extubated in 3-hour time and taken up for a pectoralis major (PM) flap. Her blood sugar remained high throughout, between 250 and $320 \mathrm{mg} \%$. Insulin infusion of 10 units over 18 hours in ICU brought the blood sugar within control as did topical vancomycin and antibiotic cover with negative pressure wound therapy (NPWT).

\section{Discussion}

Deep sternal wound infection (DSWI) following major cardiac surgery is a deleterious complication with sequelae that can be life threatening. As reported, the incidence of DSWIs range from 1 to $3 \%$, with an associated mortality rate of 19 to $29 \%{ }^{1}$

Anesthesia for DSWI should not be taken lightly. Airway control is paramount, whether done using a noninvasive ventilation face mask, laryngeal mask airway, or an ETT. Patient safety has to be ensured in terms of (i) antibiotic prophylaxis for 48 hours, (ii) glycemic monitoring and control up to 72 hours, and (iii) airway control till patient is hemodynamically stable.

For sternal wound infection (SWI), as per the The National Institute for Health and Care Excellence (NICE) guidelines, 2008, DSWI divided management into three phases: preoperative, intraoperative, and postoperative. During preoperative management, IV antibiotic prophylaxis should be given to patients undergoing surgery on a dirty or infected wound. Intraoperatively, the management of SWI involves wound dressings to cover surgical incisions with an appropriate interactive dressing, antiseptic skin preparation, and maintenance of patient homeostasis. The management of SWI postoperatively is dependent on the classification of the infection, the patient's clinical status, and the organisms that are detected in the cultures. ${ }^{2,3}$

\section{The Management of Deep Sternal Wound Infection}

The literature generally reports coagulase-negative Staphylococci and Staphylococcus aureus to be the most common pathogens isolated in DSWI. Gram-negative rods are more common in infections that persist following timely debridement and implementation of IV antibiotic therapy, and are associated with worse prognosis. Once the diagnosis is suspected, empirical broad-spectrum IV antibiotics should be initiated. Once culture results are available, antibiotics should be targeted following microbiology advice. ${ }^{4,5}$ Patients benefit from plastic surgery consultation and reconstruction as early as possible. A plastic surgeon should be present at the time of initial debridement following diagnosis of DSWI (-Figs. 1-3).

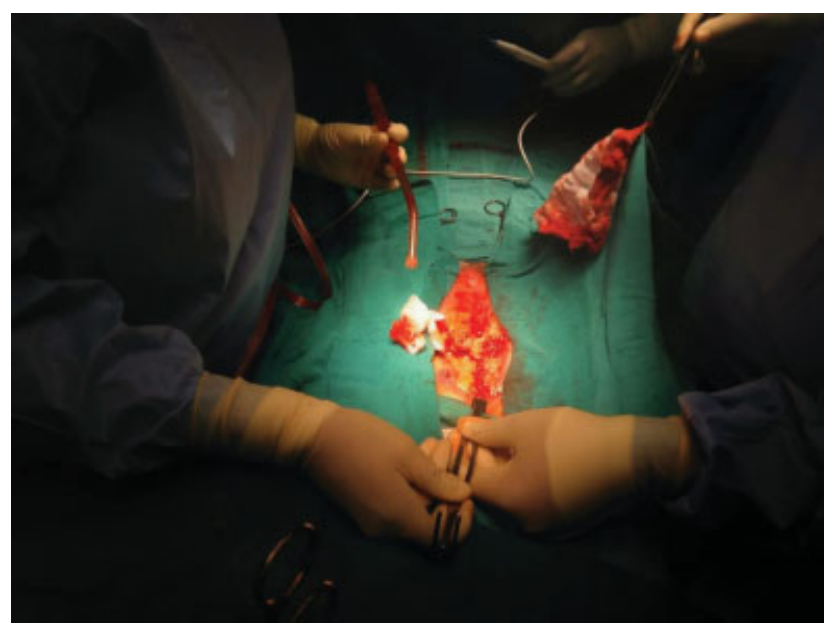

Fig. 1 Infected sternal wound at sternotomy.

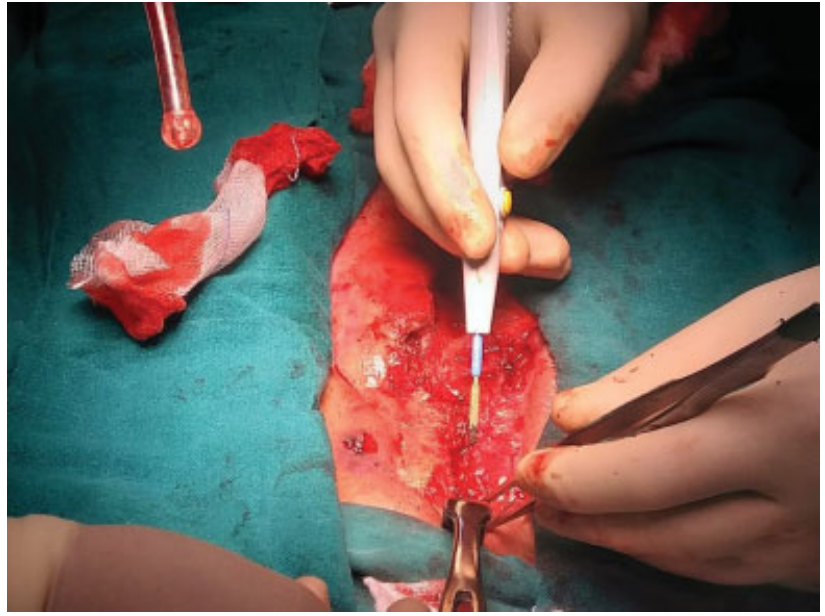

Fig. 2 Infected sternal wound debridement in situ.

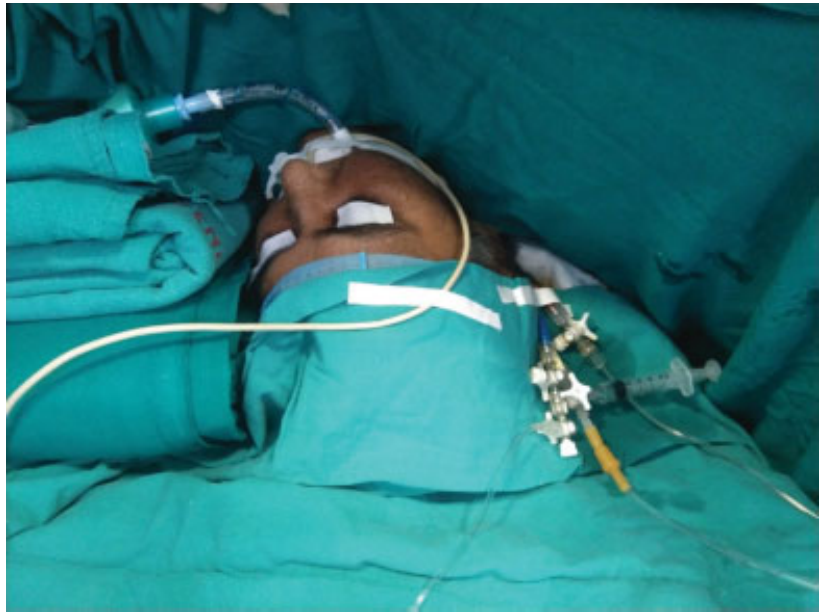

Fig. 3 Patient anesthetized with a ProSeal LMA mask on the operating table. 

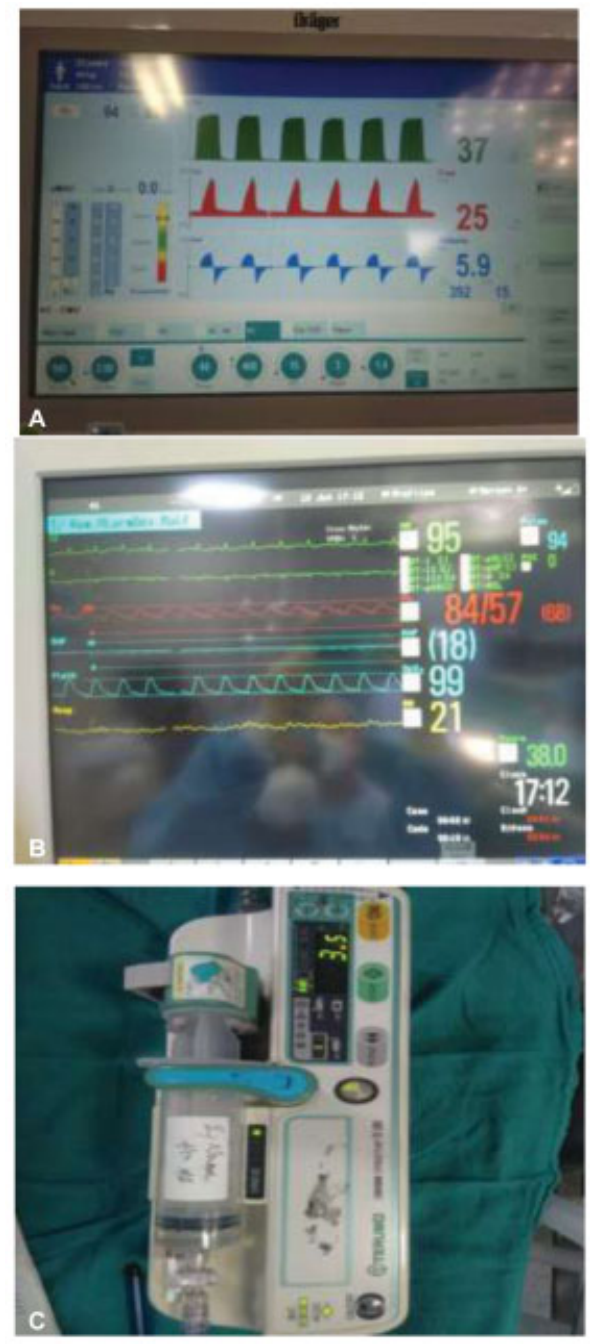

Fig. 4 (A) Mechanical ventilator during pectoralis major flap reconstruction perioperative when blood pressures came down on the monitor. (B) Monitor screen. (C) Noradrenaline injection started in intensive care unit with endotracheal tube in situ, whereas intraoperative there was no inotrope sepsis in ICU warrants starting noradrenaline.

\section{Negative Pressure Wound Therapy (NPWT)}

Many studies have been reported, which provide level 3 evidence for the role of NPWT in the management of DSWI. In a 12-year review, Lonie et al demonstrated NPWT to be associated with a reduced need for flap reconstruction and fewer postoperative complications requiring reoperation after definitive closure. None of the patients in their study
Table 2 Multivariate risk factors for deep sternal wound infections $(n=28)$

\begin{tabular}{|l|}
\hline Risk factors prolonged \\
\hline Mechanical ventilation \\
\hline Insulin-dependent diabetes \\
\hline Peripheral vascular disease \\
\hline BIMA used \\
\hline No. of grafts $>2$ \\
\hline BMI $\geq 30 \mathrm{~kg} / \mathrm{m}^{2}$ \\
\hline Reexploration for bleeding \\
\hline NYHA class $\geq 3$ \\
\hline
\end{tabular}

Abbreviations: BIMA, bilateral internal mammary artery; BMI, body mass index; NYHA, New York Heart Association.

treated with NPWT required rewiring of the sternum, suggesting stabilization of the sternum as an additional benefit. ${ }^{6}$ NPWT dressing changes should be performed in theater every 3 to 4 days, and flap coverage performed as soon as the patient is suitably stable.

\section{Early Flap Closure Is the Key to Success}

Early flap is the key to prevent multiple sutures. The aim in management of DSWI should be for early flap closure. If flap coverage is not possible at initial debridement due to instability of the patient, then-and only then-NPWT should be used alongside aggressive IV antibiotic therapy for interval wound therapy, with plan for delayed closure (-Fig. 4).

\section{Pectoralis Major Flap}

The PM flap is a reliable flap for superior $2 / 3$ sternal defects. It can be used in the absence of the internal mammary artery as an advancement flap, or as a perforator flap where the Internal Mammary Artery (IMA) is present to increase its excursion for the reconstruction of larger defects.

\section{Rule Out All Risk Factors Causing DSWI}

The incidence of DSWI in cardiac surgery is $2.0 \%$. Independent risk factors for DSWI were emergent surgery, combined surgery, and postoperative intra-aortic balloon pump (IABP) use. Systematic measures have to be taken for patients in poor condition to prevent DSWI. ${ }^{3}$ Risk factors for DSWI repeat closure should not be ignored. ${ }^{7}$

Table 1 Evidence-based recommendations by Hever et al $^{9}$

- The aim in management of deep sternal wound infection should be for early flap closure. If flap coverage is not possible at initial debridement due to instability of the patient, then-and only then-negative pressure wound therapy (NPWT) should be used alongside aggressive intravenous antibiotic therapy for interval wound therapy, with plan for delayed closure.

- NPWT dressing changes should be performed in theater every 3-4 days, and flap coverage performed as soon as the patient is suitably stable.

- The best flap option immediately available should be used, avoiding any unnecessary additional surgical incisions.

- The pectoralis major flap is a reliable flap for superior $2 / 3$ sternal defects. It can be used in the absence of the IMA as an advancement flap, or as a perforator flap where the IMA is present to increase its excursion for the reconstruction of larger defects. 
Table 3 Preventive noninvasive ventilation and sepsis management in deep sternal wound infections

- Preoperative bathing with chlorhexidine; intranasal mupirocin within 24 hours of surgery, continued for 5 days in all patients with positive staphylococcal nasal swab

- Preoperative glycemic control with insulin sliding scale

- Smoking cessation and pulmonary function optimization

- Intraoperative continuous insulin infusion to maintain serum glucose level $<6 \mathrm{mmol}$

- Topical antibiotics (vancomycin or gentamicin) applied to the cut edges of the sternum upon opening and before closing; vancomycin paste for bone marrow should replace bone wax

- Early extubation and removal of drains and urinary and central venous catheters

- Maintain airway patency and saturation with laryngeal mask airway or endotracheal tube for short duration; only fast track extubation

- Strict preoperative, perioperative, and postoperative antibiotics up to 48 hours

- Exclude risk factors like diabetes mellitus, obesity, chronic obstructive pulmonary disease, and peripheral artery disease

- Preoperative optimization of glucose control is recommended in all patients with elevated hemoglobin A1C levels ( $>7.5 \%$ and serum glucose levels ( $>200 \mathrm{mg} / \mathrm{dL}$ ), and postoperative optimization by means of continuous insulin infusion in the intensive care unit (target serum glucose $<180 \mathrm{mg} / \mathrm{dL}$ ) for at least 24 hours. At our institution, we use insulin to regulate blood glucose in this manner in all cardiac surgery patients regardless of diabetic status, given the evidence that elevated serum glucose increases the risk of sternal wound infection ${ }^{10}$

- Noninvasive ventilation approach

\section{Summary}

As DSWI leads to short- and long-term mortality, risk factors for preventing DSWI should be ruled out by the anesthesiologists. Most of these risk factors are modifiable. It is an anesthesiologist's role to prevent them perioperatively. Some of them are listed in -Table 1. So, prevent all risk factors by overcoming the challenges and adapting a definitive team with discussed protocol ( - Table 2 ). ${ }^{8}$

Summary of the key factors for favorable outcomes is presented in - Table 3 .

\section{Conclusion}

Anesthesiologist's role in ensuring risk factors takes into account giving perioperative and postoperative antibiotics, maintaining a normoglycemic state with stable hemodynamics, ensuring stability during NPWT $(\mathrm{HbA} 1 \mathrm{C}<8$ at all times), and maintaining a safe airway despite repeat sternal debridements.

Discussion with the plastic surgery team should take place as soon as diagnosis is made to enable surgical planning with consideration for early flap closure, with or without sternal plating. Where NPWT is used, this should be used for as short a period as possible, and plastic surgery coverage performed as early as possible.

Conflict of Interest

None.

\section{References}

1 Milano CA, Kesler K, Archibald N, Sexton DJ, Jones RH. Mediastinitis after coronary artery bypass grafting surgery. Circulation 1995;92:2245-2251

2 El Oakley RM, Wright JE. Postoperative mediastinitis: classification and management. Ann Thorac Surg 1996;61(03):1030-1036

3 Leaper D, Burman-Roy S, Palanca A, et al; Guideline Development Group. Prevention and treatment of surgical site infection: summary of NICE guidance. BMJ 2008;337:a1924

4 Chan M, Yusuf E, Giulieri S, et al. A retrospective study of deep sternal wound infections: clinical and microbiological characteristics, treatment, and risk factors for complications. Diagn Microbiol Infect Dis 2016;84(03):261-265

5 Charbonneau H, Maillet JM, Faron M, et al. Mediastinitis due to Gram-negative bacteria is associated with increased mortality. Clin Microbiol Infect 2014;20(03):0197-0202

6 Lonie S, Hallam J, Yii M, et al. Changes in the management of deep sternal wound infections: a 12-year review. ANZ J Surg 2015;85 (11):878-881

7 Lemaignen A, Birgand G, Ghodhbane W, et al. Sternal wound infection after cardiac surgery: incidence and risk factors according to clinical presentation. Clin Microbiol Infect 2015;21(07): 674.e11-674.e18

8 Lu JC, Grayson AD, Jha P, Srinivasan AK, Fabri BM. Risk factors for sternal wound infection and mid-term survival following coronary artery bypass surgery. Eur J Cardiothorac Surg 2003;23(06): 943-949

9 Hever P, Singh P, Eiben I, Eiben P, Nikkhah D. The management of deep sternal wound infection: literature review and reconstructive algorithm. JPRAS Open 2021;28:77-89

10 Al-Ebrahim KE. Management of deep sternal wound infection: complete sternal osteomyelitis. Heart Surg Forum 2020;23(03): E281-E284 